\title{
Prácticas matemáticas democráticas: Análisis de una experiencia escolar
}

\author{
Yuly Vanegas, Universitat de Lleida (España) \\ Joaquín Giménez, Universitat de Barcelona (España)
}

\section{Prácticas matemáticas democráticas: Análisis de una experiencia escolar}

Resumen

En este artículo proponemos caracterizar las prácticas matemáticas democráticas promovidas en una clase de matemáticas con estudiantes de 12 y 13 años centrada en la enseñanza y el aprendizaje de la medida de longitud. Se muestra que la promoción de estas prácticas posibilita negociar exitosamente significados matemáticos. Se realiza un análisis donde junto con la identificación de prácticas matemáticas democráticas se observan normas, para describir e interpretar los procesos interactivos que dan forma a la participación de profesor y estudiantes en las sesiones de clase. Los resultados indican el dominio de prácticas relacionadas con la articulación y resolución de conflictos. También se constata un determinado tipo de normas en relación con cada tipo de práctica matemática democrática.

Palabras clave. Prácticas matemáticas democráticas; normas; de aula construcción de significado; medida de longitud.

Democratic mathematical practices: Analysis of a school experience

\section{Abstract}

In this article we propose the characterization of the democratic mathematical practices promoted in a mathematics classroom with 12-13-year-old students focused on the teaching and learning of the length measurement. It is shown that the promotion of such practices makes it possible to successfully negotiate mathematical meanings. An analysis is carried out in which, in addition to the identification of democratic mathematical practices, we observe norms to describe and interpret the interactive processes that shape the participation of teacher and students in the lessons. The results indicate a predominance of practices related to the articulation and resolution of conflicts. It is also found that each type of democratic mathematical practice relates to a certain type of norm.

Keywords. Democratic mathematical practices; classroom norms; meaning construction; length measure.

\section{Introducción}

Un reto de la formación escolar actual es construir ambientes de aprendizaje que fomenten el desarrollo de competencias. Dichas competencias han de posibilitar que los estudiantes devengan ciudadanos críticos. Se resalta así la necesidad de formar sujetos con capacidad para pensar, enfrentar desafíos, tomar decisiones, adaptarse al cambio y que consideren que sus acciones pueden transformar y mejorar la sociedad (Giroux \& McLaren, 1989). Si bien son planteamientos desde una perspectiva general y global, la educación matemática no puede ser ajena. Se debe reconocer cómo enseñar y aprender matemáticas pueden responder a estos retos y contribuir a lograr los objetivos amplios de la educación (D’Ambrosio, 1990; Skovsmose, 1999).

La formación escolar debe favorecer una construcción colaborativa de significados y facilitar el desarrollo de una alfabetización matemática que permita ejercer una competencia democrática. En Darling-Hammond (1996), el desarrollo de esta competencia implica aprender a vivir en el aula y educar para la democracia. Una clase 
democrática involucra cuatro rasgos distintivos: a) un currículo basado en la resolución de problemas; b) una cultura de la inclusión y los derechos; c) una participación igualitaria en la toma de decisiones; d) la generación de oportunidades y estímulos para que todos aprendan matemáticas de forma exitosa (Ellis \& Malloy, 2007).

Se requiere revalorizar la construcción de un sentido ético de la responsabilidad profesional del docente de matemáticas y realizar estudios orientados al análisis de aspectos socioculturales y políticos que influyen o se generan en clase de matemáticas (Planas \& Valero, 2016). Investigaciones como Colomina, Onrubia y Rochera (2001) identifican la evolución favorable de mecanismos de influencia educativa como la construcción progresiva de sistemas de significados compartidos y el traspaso de control y responsabilidad en el aprendizaje del profesor a los estudiantes, cuando se propicia un ambiente cooperativo. Walshaw y Anthony (2006) identifican el poder del discurso y de los discursos hegemónicos de poder en las relaciones de aula. Planas y Edo (2008) reconocen además procesos de construcción de significados matemáticos emergentes del análisis de la participación en clase de matemáticas y del discurso del profesor en la generación de oportunidades de aprendizaje.

En este artículo proponemos, por una parte, caracterizar las prácticas matemáticas democráticas promovidas en una clase de matemáticas centrada en la medida de longitud. Por otra parte, mostramos que la promoción de dichas prácticas posibilita negociar exitosamente significados matemáticos. Para ello, se realiza un análisis donde junto con la identificación de prácticas matemáticas democráticas se observan normas, que regulan las interacciones y el funcionamiento social de la clase.

\section{Marco teórico}

Las prácticas sociales de la educación matemática no pueden aprenderse sin considerar los sistemas de creencias sobre las matemáticas, su enseñanza y aprendizaje. Tal y como plantea Valero (2019), estos sistemas dan cuenta de la naturaleza y normatividad de la educación matemática. Hacer una aproximación a la clase de matemáticas como microsociedad y estudiar las reglas sociales que la caracterizan implica analizar la lengua y la participación. Según Lantolf, Thorne y Poehner (2015), estos aspectos (lengua y participación) son instrumentos mediadores del desarrollo cognitivo por excelencia. Por lo tanto, para construir el conocimiento de forma sistemática y eficaz es necesaria una participación dialogada, la cual incorpora el contraste y el conflicto. Se busca así que los participantes se sientan implicados al ser escuchados e involucrados como miembros de un grupo. En consecuencia, se requiere generar escenarios en los que se interactúa compartiendo deberes y responsabilidades (Engeström, 1994); y en los que se co-construyan significados (Crook, 1994) a través de diálogos deliberativos (Serradó, Vanegas \& Giménez, 2015).

Consideramos como D'Ambrosio (2011) que desarrollar un ambiente en clase de matemáticas en el que se asumen los planteamientos señalados conlleva un nuevo papel del profesor, que le exige asumir responsabilidad en la transformación de un currículo tecnocrático hacia uno dinámico y creativo que incorpore una dimensión ética de la educación. Esta dimensión considera que la responsabilidad de matemáticos y educadores es saber cómo orientar un pensamiento crítico a través del discurso igualitario (Freire, 1988) desde una perspectiva ética. D’Ambrosio (2011) alude a una ética para la diversidad que promueve la comprensión, la reflexividad y la autocrítica y se organiza mediante tres componentes: respeto, solidaridad y colaboración. El respeto se entiende como la consideración de las diferencias individuales. La solidaridad se 
entiende como la satisfacción de las necesidades básicas de supervivencia de los individuos. Finalmente, la colaboración aquí se entiende en el sentido de preservar el patrimonio cultural y natural común, no como diálogo o forma de discurso.

Para participar en una deliberación democrática se necesitan ciertas habilidades, actitudes y valores. De acuerdo con Habermas (1998), un buen proceso deliberativo se basa en una situación comunicativa en la cual todos pueden contribuir, todos tienen una misma voz y pueden hablar libremente y honestamente sin restricciones (Chappell, 2012). El dialogo deliberativo generalmente se estructura en torno a una pregunta lo bastante abierta para permitir un desacuerdo, pero al mismo tiempo lo bastante cerrada para dirigir la discusión hacia una conclusión (Samuelsson, 2016).

Una situación educativa democrática deliberativa, según Englund (2006), es aquella en la que: se confrontan diferentes puntos de vista y se articulan argumentos a favor de los mismos; hay tolerancia y respeto por el otro y los participantes escuchan los argumentos de los demás; hay elementos de formación de una voluntad colectiva, un deseo de alcanzar un consenso o un acuerdo temporal. Una práctica democrática deliberativa debe permitir el cuestionamiento de las tradiciones y buscar que la comunicación deliberativa se produzca finalmente sin el control del profesor.

Skovsmose y Valero (2012) señalan que es fundamental trabajar por un acceso democrático a la educación matemática, es decir, por una educación que favorezca la consolidación de relaciones sociales democráticas en los diferentes terrenos en los que tienen lugar las prácticas de la educación matemática: aula, organización escolar y sociedad (local y global). En nuestro estudio, exploramos la consolidación de dichas relaciones sociales en el ámbito de la clase; nos interesa analizar la participación y la actividad matemática que se promueve en un ambiente en el que se considera el diálogo como proceso clave en la construcción de significados.

Cuando se realiza el análisis de la actividad matemática en un aula, podemos ver cómo aparecen en el transcurso de la clase determinadas reglas matemáticas (por ejemplo, cuando se institucionaliza una definición o un procedimiento), pero también cómo se repiten patrones de interacción que explícita o implícitamente están generando normas y valoraciones, explícitas o implícitas, de los alumnos sobre dichas normas (D’Amore, Godino, \& Font, 2007; Civil \& Planas, 2011). La cantidad de reglas que regulan la práctica matemática de los alumnos en el aula da pie a combinaciones que producen tipos de práctica matemática. Nosotros nos fijamos en las prácticas matemáticas que efectivamente permiten construir significado matemático entre quienes participan en ellas. Más concretamente, llamamos prácticas matemáticas democráticas a las que involucran la co-construcción crítica de significado matemático mediante un sistema de interacciones dialogadas en el aula (Vanegas, 2013). Para caracterizar este tipo de prácticas, en Vanegas, D’Ambrosio y Giménez (2019) se propone una tipología construida a partir del cruce de las categorías en la propuesta de la ética de la diversidad (D’Ambrosio, 2011) y en la de co-construcción crítica de significados matemáticos (Crook, 1994). Se definen nueve tipos de prácticas:

- Práctica de Articulación Respetuosa (PAR): El profesor hace preguntas abiertas y/o provocadoras, a veces interpretativas, a fin de interactuar en un diálogo igualitario con significados matemáticos de los estudiantes, quienes hacen declaraciones personales de significados, estrategias o formas de razonamiento.

- Práctica de Articulación Solidaria (PAS): El profesor hace intervenciones que buscan el contraste, a fin de que se declaren públicamente dificultades y motiva 
que sean los estudiantes quienes colaboren para superarlas. Se busca que esta colaboración vaya más allá de buscar respuesta a la tarea matemática.

- Práctica de Articulación Colaborativa (PAC): El profesor motiva que los estudiantes realicen, a nivel grupal, síntesis sobre los significados matemáticos involucrados en las tareas. Se reconoce la identidad del grupo como institución que colabora en la generación de conocimiento matemático.

- Práctica de Generación Respetuosa de Conflictos (PGRC): El profesor prevé conflictos (cognitivos, semióticos, etc.) y promueve a través de preguntas y/o tareas reconocer interpretaciones diferentes, validar argumentaciones de estudiantes, resolver desacuerdos, a fin de que el alumnado reconozca posicionamiento, reflexión y regulación como procesos clave en la construcción de significado.

- Práctica de Solidaridad ante los Conflictos (PSC): El profesor provoca que varios estudiantes expongan sus argumentos a fin de ayudar a mejorar la comprensión de otros. Se busca ganar en coherencia y profundidad, y que el alumnado reconozca la ayuda como una responsabilidad.

- Prácticas de Colaboración ante los Conflictos (PCC): El profesor anima diálogos en los que se discute y realizan acuerdos para resolver conflictos, lo que motiva la transformación de significados. Los estudiantes asumen que la construcción de significado es posible a través de la negociación de ideas con los demás.

- Práctica de Co-construcción Respetuosa (PCoR): El profesor promueve el análisis de propuestas de organización y estructuración sistémica de objetos y procesos matemáticos, y plantea tareas en las que se transfieren significados matemáticos a otros contextos. El alumnado utiliza y reflexiona sobre la importancia de usar técnicas de síntesis (esquemas, cuadros comparativos, mapas conceptuales, etc.).

- Práctica de Co-construcción Solidaria (PCoS): El profesor hace intervenciones con las que busca ayudar al grupo a clasificar y organizar información. Repite ideas del alumnado mejorando formas de expresión de acuerdos logrados sobre significados matemáticos Gestiona emociones, considerando potencialidades de cada alumno.

- Práctica de Co-construcción Colaborativa (PCoC): El profesor actúa como moderador y animador de debates dialógicos donde se comparten y verifican consensos sobre ideas matemáticas, a fin de reconocer y definir estrategias de acción común que permitan interpretar el valor social de la construcción matemática. Los estudiantes identifican la práctica matemática como conservación de la cultura matemática del grupo (patrimonio colectivo).

\section{Contexto y metodología de estudio}

Se realiza un estudio etnográfico de caso (Creswell, 1988), donde el caso es el de las prácticas del grupo humano que configura una clase de matemáticas de un instituto público de educación secundaria de Cataluña. Se busca estudiar, describir y comprender el funcionamiento social de la clase cuando los participantes se involucran en el desarrollo de tareas matemáticas. Se analiza una experiencia escolar con 25 alumnos de 12 y 13 años. La experiencia de clase está orientada al estudio de la medida de longitud. El profesor busca que se relacione la noción de medir inicialmente con la idea de comparar a fin de alejar a los estudiantes de la concepción habitual de que medir es calcular. Las primeras actividades de la secuencia se centran en la identificación de unidades arbitrarias tradicionales no decimales asociadas al cuerpo humano (palmos, codos, brazadas, etc.) y en el cambio de unidades. Las actividades siguientes se refieren al deseo europeo de llegar a una unidad de medida común para 
todos y, finalmente, a cómo se llegó a determinar la medida del metro. Se plantean actividades en las que se pretende que los alumnos reconozcan la inconveniencia del uso de instrumentos de medida con diferentes unidades de medida. Se contextualiza la situación con el momento histórico que dio lugar a la creación del metro.

Se observaron, grabaron en vídeo y analizaron cuatro sesiones de clase. Para el análisis de la experiencia se ha subdividido el registro en nueve episodios. En este artículo analizamos el episodio 6 por ser uno de los que consideramos más ricos en cuanto a la actividad matemática colectiva que se puede llegar a identificar. Ahí se discute y argumenta sobre el proceso de conmensurabilidad, que surge como respuesta a un problema abordado en un episodio anterior. En el conjunto de episodios de la experiencia se reconocen los contenidos de: a) identificar los dos componentes de una medida, unidad y número asociado al número de veces que se encuentra la unidad; b) usar comparaciones entre medidas del mismo tipo, y c) establecer una relación multiplicativa asociada al cambio de medidas con unidades diferentes.

Con los datos mencionados, se realizan dos análisis. En un primer momento se identifican las prácticas matemáticas democráticas a partir del sistema de categorías definidas en Vanegas et al. (2019), las cuales se han descrito en el apartado 2. En un segundo momento se utilizan herramientas del enfoque onto-semiótico para identificar las normas. Desde este enfoque se asume que el aprendizaje matemático está condicionado no sólo por conocimientos matemáticos y didácticos, sino también por reglas (Vanegas, Font, \& Pino-Fan, 2020). Para el análisis de la práctica matemática Godino, Font, Wilhelmi \& Castro (2009) proponen seis tipos de normas: a) Epistémicas, refieren fundamentalmente al contenido matemático, las situaciones adecuadas para su aprendizaje y las representaciones asociadas a dichos contenidos. $b$ ) Cognitivas, son aquellas que evidencian el desarrollo de significados personales (aprendizajes), permiten reconocer la manera en que los estudiantes construyen y comunican significados matemáticos. c) Interaccionales, determinan la secuencia de interacciones entre el profesor y estudiantes orientadas a la fijación y negociación de significados. d) Mediacionales, estas normas aluden a la distribución de los recursos tecnológicos utilizados y asignación del tiempo a las distintas acciones y procesos. e) Afectivas, se relacionan con la distribución temporal de actitudes, emociones, afectos, motivaciones, de cada alumno con relación a los objetos matemáticos y al proceso de estudio seguido. Y, f) Ecológicas, explican las relaciones con el entorno (sociocultural, político, económico, etc.) en el que se desarrolla el proceso de instrucción. Varias de estas normas se ajustan bien en realidad a tipos de normas ubicados en teorías socioculturales de la educación matemática considerados en Planas y Boukafri (2018).

Finalmente, se contrastan estos dos análisis, de prácticas matemáticas y de normas del aula, para enriquecer el estudio contando con más elementos que permitan identificar la construcción de significados sobre la medida de longitud que se posibilitan cuando se promueven prácticas matemáticas democráticas

\section{Análisis y resultados}

En las sesiones iniciales de la experiencia, el profesor trabaja con los estudiantes sobre tipos de unidades no convencionales para la medida de longitud que se usaban en Catalunya entre los siglos XVI y XVIII. Se introduce el patrón llamado cana, cuyo valor asignado dependía de la población. En este momento de la interacción de clase se sitúa el episodio 6 con el que ilustramos la identificación de prácticas matemáticas democráticas, de normas de aula y las relaciones entre unas y otras. 


\subsection{Análisis del episodio 6}

El episodio 6 inicia con estudiantes $\left(\mathrm{E}_{\mathrm{i}}\right)$ respondiendo a la discusión que quedó abierta en un episodio anterior donde el profesor $(\mathrm{P})$ planteó la siguiente pregunta: $S i$ 340 canas de Arenys son quinientos y pico metros y me habéis dicho que la cana es más larga que el metro, ¿cómo es posible que el número de canas sea más pequeño que el número de metros? Esta pregunta genera otros cuestionamientos con declaraciones personales distintas. Esta es la transcripción del episodio (nótese que reproducimos una versión de la transcripción con turnos consecutivos de un mismo participante separados en función de los enunciados que preliminarmente se han analizado como posibles informadores de prácticas matemáticas democráticas y/o de normas; también nótese que esta versión de la transcripción incluye algunas de nuestras interpretaciones iniciales entre corchetes):

$1 \mathrm{P} \quad$ A ver, quien quiera intervenir que levante la mano.

2 E $\quad \mathrm{E}_{\mathrm{s}} \quad$ [Varios estudiantes levantan la mano]

3 P [P da la palabra a uno de los que ha levantado la mano y pide a otro que no intervenga en ese momento]

4 P A ver tú que...

$5 \quad \mathrm{E}_{\mathrm{J}} \quad$ Es más grande la cana. [P solicita que hable más alto]

$6 \mathrm{E}_{\mathrm{J}}$ ¿Por qué es más grande la cana?

$7 \mathrm{P}$ Silencio.

8 E La cana es un metro y medio, y el metro es un metro.

$9 \quad \mathrm{E}_{\mathrm{C}} \quad$ [Indica con la mano que quiere intervenir]

$10 \mathrm{E}_{\mathrm{O}}$ ¿Y por qué tampoco es un metro y medio?

$11 \mathrm{E}_{\mathrm{C}}$ Porque un metro es un metro.

$12 \mathrm{E}_{\mathrm{C}}$ Un metro es un metro.

$13 \mathrm{P}$ ¿Qué problema tiene $E_{J}$ ?

$14 \mathrm{P}$ A ver si podemos averiguar qué problema tiene.

$15 \mathrm{E}_{\mathrm{C}}$ Ella piensa que un metro es muy diferente que la cana. [Interviene sin tener la palabra]

$16 \mathrm{P}$ Está muy bien que ocurra esto, porque nos presenta dudas que nos hacen pensar.

17 P Los otros de la clase que no lo entienden bien, están distraídos y no preguntan nada.

$18 \mathrm{P}$ Por tanto, está muy bien, nos ayuda lo que dices.

$19 \mathrm{P} \quad$ A ver quién lo explica... Inténtalo, $E_{C}$.

$20 \mathrm{E}_{\mathrm{C}}$ Ella piensa que el metro es lo que mide la cana y que será lo mismo que la cana que será un metro y medio.

$21 \mathrm{E}_{\mathrm{C}} i Y$, no! Un metro es un metro, pero la cana hace un metro y medio, a la cana le sacas medio metro y hace medio.

$22 \mathrm{P}$ Es decir, que cuando se inventó el metro, se inventó un metro que era más pequeño que la cana.

$23 \mathrm{E}_{\mathrm{C}}$ Es la unidad.

$24 \mathrm{P}$ Porque se inventó así el metro. No es que haya una razón especial. Ella lo que

dice es, ¿por qué el metro no podía medir un metro y medio?

$25 \mathrm{P}$ Claro que el metro podría medir un metro y medio.

$26 \mathrm{P}$ El metro podría haber sido mucho más largo.

$27 \mathrm{E}_{\mathrm{C}}$ Es la unidad de medida.

$28 \mathrm{P}$ El metro pueden haberlo hecho así [Extiende los brazos en forma horizontal indicando una longitud grande].

$29 \mathrm{P}$ Pero, no lo hicieron así, lo hicieron así [Busca mostrar un metro extendiendo un brazo en forma horizontal desde el hombro contrario]

$30 \quad \mathrm{E}_{\mathrm{C}} \quad$ [Cambia sus gestos, parece satisfecho de estar entendiéndolo mejor]

$31 \mathrm{P}$ El próximo día os explicaré de dónde salió este trozo. Por qué se dijo que era 

así el metro.
$32 \mathrm{P}$ No fue casual, os digo que tiene relación con lo de la tierra. Ahí está la gracia del metro.
$33 \mathrm{P} \quad$ ¿Entendéis? Pero se inventó así el metro, así de corto. No era más largo que eso.
$34 \mathrm{P}$ Se inventó de esa manera.

\section{Análisis de las prácticas matemáticas democráticas}

Para determinar las prácticas matemáticas democráticas, observamos la interacción en el episodio, en las grabaciones y su versión simplificada en la transcripción. Identificamos primero momentos donde profesor y estudiantes intervienen en la producción de ideas matemáticas. A dichos momentos se asocian prácticas según la tipología comentada, con especial atención al contenido matemático que vehicula el intercambio de intervenciones. Como se observa en la Tabla 1, en el episodio 6 identificamos hasta cinco tipos de práctica matemática democrática, resaltando aquellas cuyo foco está en el planteamiento y resolución de conflictos entre significados matemáticos vinculados con la tarea o pregunta que se está discutiendo.

Tabla 1. Tipos de práctica matemática democrática en el episodio 6

\begin{tabular}{|c|c|c|}
\hline & Prácticas matemáticas democráticas & $\mathbf{P} / \mathbf{E}$ \\
\hline PAR & $\begin{array}{l}\text { Organiza la forma de intervenir para que haya participación igualitaria }[1,3,4] \text {. } \\
\text { Participan para exponer ideas individuales sobre la tarea }[2,5] . \\
\text { Reconocen que puede haber respuestas distintas a la tarea }[5]\end{array}$ & $\mathrm{P}$ \\
\hline PGRC & $\begin{array}{l}\text { Plantean dudas de forma reflexiva (¿Por qué es más grande la cana? ¿Y por } \\
\text { qué tampoco es un metro y medio?) [6,10] } \\
\text { Pide atención para que todos escuchen y se centren en la duda formulada [7]. } \\
\text { Intervienen para ayudar a resolver interpretaciones diferentes y mostrar su } \\
\text { posicionamiento sobre la medida de la cana, el metro y las relaciones entre } \\
\text { estas dos medidas }[8,9,11,12] \text {. } \\
\text { Establecen la relación entre el número y la medida }[11,12] \text {. }\end{array}$ & $\mathrm{E}$ \\
\hline PSC & $\begin{array}{l}\text { Invita a pensar en la duda de una estudiante }[13,14] \text {. } \\
\text { Resalta la importancia de la duda ya que plantea un razonamiento más allá de } \\
\text { buscar la respuesta a la tarea (comparación entre dos medidas) }[16,18] \text {. } \\
\text { Interviene buscando mejorar la comprensión sobre la duda de la compañera, } \\
\text { resaltando aspectos clave }[15] \text {. } \\
\text { Asume la duda de una alumna como tarea matemática para todos }[16,17,18] \text {. }\end{array}$ & $\mathrm{P}$ \\
\hline PCC & $\begin{array}{l}\text { Solicita participación para explicar la pregunta/duda de una compañera [19] } \\
\text { Interviene para exponer explicaciones/argumentaciones a la duda de una } \\
\text { compañera que se asume como pregunta de la clase [20,21]. } \\
\text { Interviene para centrar un aspecto relevante en la comprensión de la } \\
\text { construcción de la unidad de medida convencional [22]. } \\
\text { Reformula la duda planteada por la alumna, para remarcar la importancia de la } \\
\text { pregunta en la comprensión de cómo llegar a una unidad convencional [24]. } \\
\text { Hacen ampliaciones a respuestas de alumnado que ayudan a transformar o } \\
\text { consolidar significados }[25,26,28,29] \text {. } \\
\text { Hacen proposiciones que evidencian comprensión de las "nuevas" relaciones } \\
\text { matemáticas reconocidas }[23,27] \text {. }\end{array}$ & $\mathrm{P}$ \\
\hline $\mathrm{PCoS}$ & $\begin{array}{l}\text { Manifiestan satisfacción al ver reconocidas sus intervenciones y entender las } \\
\text { conclusiones surgidas del diálogo [30]. } \\
\text { Recapitula ideas clave y anticipa un objetivo de trabajo }[31,32,33,34] \text {. } \\
\text { Busca motivar anticipando argumentos sobre los acuerdos a los que se llegó } \\
\text { para la construcción del metro, con conexiones entre matemáticas e historia } \\
{[31,32,33,34] \text {. }}\end{array}$ & $\mathrm{E}$ \\
\hline
\end{tabular}




\section{Análisis de las normas}

Ahora se analiza el episodio usando herramientas del enfoque onto-semiótico relacionadas con el aspecto normativo (Godino, et al., 2009). Se identifican los tipos de norma en el ambiente de clase (Tabla 2), distinguiéndose en el propio enunciado según convenga quien regula la actividad, profesor o estudiantes. Se constata que en este episodio aparecen normas de cada uno de los tipos considerados: meta-epistémico, cognitivo, interaccional, mediacional, afectivo y ecológico.

Tabla 4. Tipos de norma en el episodio 6

\section{Meta-epistémicas}

N1: Hay que atender cuando se formula una pregunta/actividad [17]

N2: Argumentar en clase de matemáticas es importante, permite analizar y concluir cuando son diferentes las respuestas a una pregunta $[19,24]$

N3: Es importante reafirmar las respuestas/preguntas dadas por estudiantes para construir significados [16, 18, 22]

N5: Es importante explorar las comprensiones de los estudiantes [13, 14]

N6: Argumentar ayuda a relacionar significados [22, 25, 26, 28]

\section{Cognitivas}

N7: Se deben reconocer y valorar públicamente dificultades/dudas de estudiantes [16]

N8: Se requieren aclaraciones sobre significados matemáticos relacionados con dificultades del alumnado [22, 24]

\section{Interactivas}

N9: Se debe intervenir con orden cuando se discute una tarea [1, 3, 4]

N10: $P$ interviene para solicitar explicaciones de respuestas de estudiantes $[4,19]$

N11: Es importante escuchar las explicaciones de compañeros $[1,3,7]$

N12: P ayuda con preguntas intermedias a mejorar explicaciones [13, 14]

N13: Se debe repreguntar para centrar la atención de la clase ante una pregunta clave [14, $16,18]$

\section{Mediacionales}

N14: Se puede usar la lengua de gestos como representación para ayudar a la comprensión de ideas matemáticas $[28,29]$

\section{Afectivas}

N15: $\mathrm{P}$ participa de varias formas para motivar [31, 32, 33, 34]

\section{Ecológicas}

N16: Incorporar elementos de la historia de las ideas científicas es relevante para su comprensión y contextualización [31, 32, 33]

N17: Se pueden establecer relaciones temporales para preparar al alumnado sobre nuevos significados o ideas matemáticas a trabajar [31]

\section{Meta-epistémicas}

N18: Los estudiantes responden a tareas que $P$ plantea $[5,15,20]$

N19: Los estudiantes intervienen afirmando o refutando afirmaciones de compañeros, para mejorar la comprensión de nociones [11, 12, 20,21]

N20: Es importante expresar argumentos que muestran significados más amplios [23, 27]

\section{Cognitivas}

N21: Los estudiantes pueden formular libremente dudas/preguntas que contribuyen al desarrollo de significados $[6,10]$

N22: Los estudiantes intervienen para responder a dudas/preguntas de otros $[8,11,12]$

N23: Los estudiantes intervienen con argumentaciones cada vez más completas con las que mejorar la comprensión de los otros [15, 20,21]

\section{Interactivas:}

N24: Los estudiantes asumen la forma de intervención acordada $[2,9]$

N25: Los estudiantes intervienen espontáneamente ante preguntas de clase $[6,15]$

N26: Es importante atender a explicaciones/preguntas de compañeros [9] 
N27: Es fundamental intervenir en buscar consensos sobre la tarea matemática [15, 20, 21]

Afectivas

N28: Los estudiantes a través de gestos o expresiones muestran su motivación cuando reconocen que lo que han explicado, lo entiende otro compañero y $\mathrm{P}$ lo valida [30]

Respecto a la actividad matemática en el episodio 6, se insiste en la medida como comparación. El diálogo se centra en cómo una medida se asocia con un número y se refiere al tamaño de un objeto tomando como referencia una unidad convencional. Se produce un conflicto al comparar las dos medidas ya que la unidad convencional (metro) es menor a la otra medida (cana), además considerando que la cana es un instrumento que aparece históricamente antes que el metro. Precisamente porque se explica la relación cana-metro con un número mayor de uno, es difícil para algunos estudiantes ver que eso implica que la medida del metro es menor. Se evidencia también que se activan diversos procesos matemáticos de comunicación, de razonamiento y de argumentación, entre otros.

\subsection{Relaciones entre prácticas matemáticas democráticas y normas}

Para enriquecer la interpretación sobre el funcionamiento social de la clase y la práctica matemática y así contar con más elementos que permitan identificar la construcción de significado que se posibilita cuando se promueven prácticas matemáticas democráticas, se exploran relaciones entre los tipos de norma y los tipos de práctica matemática democrática identificados en el episodio 6. A fin de visualizar de manera global estos análisis, la Figura 1 presenta una sistematización integrada de los principales aspectos identificados para este episodio. El episodio se ha subdividido en agrupaciones de líneas que indican momentos donde predomina un tipo de práctica matemática democrática, en concordancia con el método de segmentación de turnos en la transcripción mostrada en este artículo.

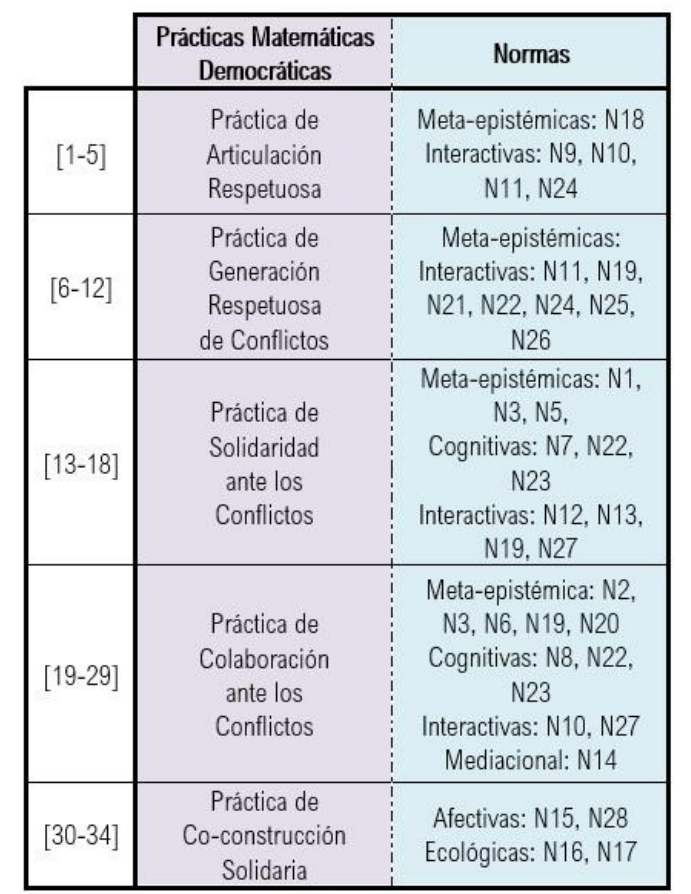

Figura 1. Cuadro integrador para el episodio 6

El cuadro integrador permite hacer diferentes lecturas. Por una parte, una lectura horizontal posibilita reconocer correspondencias entre tipos de práctica matemática democrática y tipos de norma, además de concretar unos y otros tipos a partir de los 
análisis resumidos en apartados anteriores. Por otra parte, una lectura vertical, además de posibilitar analizar cada aspecto de manera independiente, permite ver el desarrollo temporal de prácticas matemáticas democráticas. Se observa que los alumnos participan en la construcción de significados matemáticos involucrados en las tareas propuestas. Esta forma dialógica/democrática de proceder, promovida por el profesor, se ha desarrollado en un ambiente de clase soportado por un entramado diverso de normas. Dicho entramado es asumido por los estudiantes y ha contribuido a que ellos contribuyan a la construcción de ideas matemáticas.

Un resultado del análisis integrado es que a un determinado tipo de práctica matemática democrática se asocia un tipo de norma. Por ejemplo, una práctica de articulación respetuosa se relaciona fundamentalmente con normas meta-epistémicas e interactivas: "Los estudiantes construyen respuestas a tareas que se plantean en clase" (N10) y "Los estudiantes intervienen espontáneamente a preguntas en clase" (N11). Esto es consistente con nuestras nociones de articulación y respeto, que enfatizan la importancia de involucrar al alumnado en un diálogo igualitario, donde los participantes exponen sus ideas públicamente y se asume que estas ideas pueden ser diferentes. Para implicar a los estudiantes en estos procesos, conviene que el profesor considere el carácter de las tareas matemáticas que propone. En este caso se observa cómo el profesor reconoce la importancia de la contextualización y por ello introduce elementos históricos en la formulación, análisis y discusión de las tareas matemáticas. Con esto, el profesor motiva al alumnado y hace notar cómo la matemática puede operar como fuente de poder en el sentido de Skovsmose y Valero (2002).

Las prácticas de colaboración ante conflictos, en las que se resalta la importancia de la construcción colectiva de significado matemático y la síntesis de ideas clave en la resolución de preguntas y/o tareas, se asocian con una gran variedad de normas. Hay normas meta-epistémicas ("Argumentar en clase de matemáticas es importante, permite analizar y concluir cuando son diferentes las respuestas a una pregunta" N2); cognitivas ("Los estudiantes intervienen con argumentaciones cada vez más completas con las que mejoran la comprensión de los otros" N23); interactivas ("Es fundamental intervenir en buscar consensos sobre la tarea matemática" N27); y mediacionales ("Se puede usar la lengua de gestos como representación para ayudar a la comprensión de ideas matemáticas" N14).

Las prácticas de co-construcción solidaria se relacionan fundamentalmente con normas de tipo afectivo y ecológico, por ejemplo: "Los estudiantes a través de gestos o expresiones muestran su motivación cuando reconocen que lo que han explicado, lo entiende otro compañero y P lo valida" (N28) e "Incorporar elementos de la historia de las ideas científicas es relevante para su comprensión y contextualización" (N16). Es clave en este tipo de práctica que el profesor repita ideas y ayude los estudiantes a mejorar formas de comunicación de ideas matemáticas y gestione aspectos emocionales. En los episodios analizados se considera la contextualización como proceso clave en la significación de ideas matemáticas, junto con las intervenciones para declarar conexiones entre lo abordado en sesiones anteriores y futuras.

Tanto las prácticas matemáticas democráticas como las normas identificadas en el episodio analizado, implican acciones de estudiantes y del profesor, quienes participan a lo largo de las sesiones de clase en procesos de interacción dialógica. Hemos encontrado cinco tipos de práctica matemática democrática. Aunque no podemos generalizar, pensamos que, si en la clase de matemáticas se considera el diálogo y la 
participación como procesos fundamentales en la construcción del conocimiento matemático, habrá un inicio con prácticas de articulación respetuosa.

Más en general, el análisis integrado permite una interpretación más completa del funcionamiento social de la clase y de su influencia en la producción y transformación de significados matemáticos. En la Figura 2 representamos cómo se construye la práctica matemática a través de un proceso cíclico en el que prácticas matemáticas democráticas y normas generan un entramado que determina el funcionamiento social de la clase posibilitando la transformación de significados matemáticos.

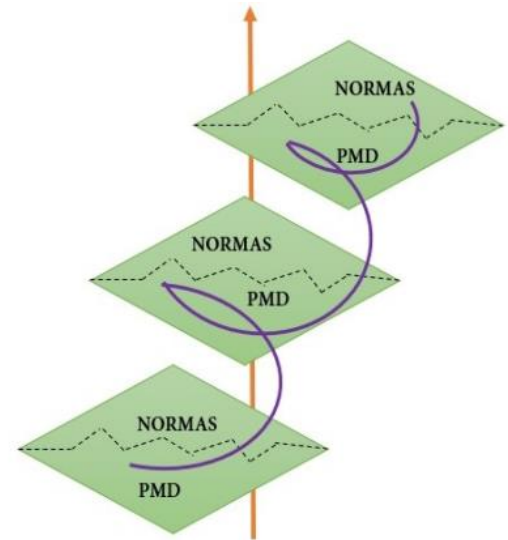

Figura 2. Papel de prácticas matemáticas democráticas (PMD) y normas en la construcción de conocimiento matemático

\section{Reflexiones finales}

En las últimas décadas ha habido un incremento en el interés por el análisis de aspectos socioculturales de la clase de matemáticas. Sin embargo, hay un gran camino por recorrer para interpretar mejor el funcionamiento social de esta clase y la influencia de los aspectos socioculturales en la construcción de conocimiento matemático. En este sentido, el estudio presentado combinando análisis desde los planteamientos de la ética de la diversidad (D'Ambrosio, 2011; Vanegas et al., 2019) y usando el constructo de normas de Godino et al. (2009) ha permitido caracterizar con riqueza el ambiente social de la clase estableciendo relaciones entre prácticas matemáticas democráticas y normas, así como la influencia de prácticas y de normas y de las relaciones entre ambas en la construcción de significado matemático.

Si bien no todos los nueve tipos de práctica matemática democrática han sido identificados en el episodio ilustrado, en el inicio de las sesiones de clase analizadas son fundamentales las prácticas de articulación, mientras que se identifican prácticas más relacionadas con la co-construcción y la definición de conflictos, cuando se producen momentos de argumentación. La resolución de estos conflictos estimula el razonamiento, la crítica y la apropiación de nuevas ideas. Tal y como lo plantea Planas (2017), la actividad matemática que se promueve en este tipo de práctica, genera oportunidades de aprendizaje matemático relevantes en la construcción de significado. Por otra parte, nuestros resultados están en la línea de Valero (2019), cuando afirma que la construcción de relaciones democráticas en la clase de matemáticas implica interacción donde los participantes se comprometen con los demás y con su propia actividad y se asumen responsables de la matemática construida de forma colectiva en clase. Además, las prácticas matemáticas democráticas brindan a los estudiantes la oportunidad de participar en situaciones educativas de deliberación en el sentido de Englund (2006), ya que fomentan razonamiento, reflexión y consenso. Procesos que 
este autor señala como requisitos para que los estudiantes sean cada vez más competentes en la deliberación democrática. En el ámbito de la educación para la democracia deliberativa existe un acuerdo general de que los futuros ciudadanos aprenden habilidades y valores para la participación democrática deliberativa principalmente participando en situaciones deliberativas, y no, por ejemplo, leyendo sobre democracia deliberativa (Samuelsson, 2016).

La experiencia escolar descrita junto con los análisis realizados es un ejemplo a seguir por docentes y futuros docentes. No sólo por la actividad matemática generada a partir de razonamientos, reformulaciones y el establecimiento de tareas contextualizadas involucrando aspectos históricos (visión de las matemáticas en conexión con la sociedad), sino porque el propio ambiente construido brinda herramientas al alumnado para desenvolverse como ciudadanos (que saben participar en diálogos deliberativos) dentro y fuera del entorno escolar.

\section{Agradecimientos}

Trabajo en colaboración con los equipos de PID2019-104964GB-100 y PGC2018098603-B-I00 (MICINN); y los equipos de SGR-2017-101 y SGR-2017-1181.

\section{Referencias}

Chappell, Z. (2012). Deliberative democracy: A critical introduction. Nueva York: Palgrave Macmillan.

Civil, M., \& Planas, N. (2011). Language policy and the teaching and learning of mathematics. En M. Setati, T. Nkambule, \& L. Goosen (Eds.), Proceedings of the ICMI Study 21 Conference on Mathematics Education and Language Diversity (pp. 38-45). Sao Paulo, Brasil: ICMI.

Colomina, R., Onrubia, J., \& Rochera, M. J. (2001). Interactividad, mecanismos de influencia educativa y construcción del conocimiento en el aula. En C. Coll, J. Palacios, \& A. Marchesi (Eds.), Desarrollo psicológico y educación, 2. Psicología de la educación escolar (pp. 437-458). Madrid: Alianza.

Creswell, J. (1998). Qualitative inquiry and research design. Choosing among five traditions. Thousand Oaks, CA: Sage.

Crook, C. (1994). Computers and the collaborative experience of learning. Londres, Inglaterra: Routledge.

D'Ambrosio, U. (1990). The role of mathematics education in building a democratic and just society. For the Learning of Mathematics, 10(3), 20-23.

D’Ambrosio, U. (2011). Educação para uma sociedade em transição. Porto Alegre, Brasil: Cortez.

D’Amore, B., Godino, J., \& Font, V. (2007). La dimensión metadidáctica en los procesos de enseñanza y aprendizaje de la matemática. Paradigma, 28(2), 49-77.

Darling-Hammond, L. (1996). The right to learn and the advancement of teaching: Research, policy, and practice for democratic education. Educational Researcher, 25(6), 5-17.

Ellis, M., \& Malloy, C. E. (2007). Preparing teachers for democratic mathematics education. En D. Pugalee, A. Rogerson, \& A. Schinck (Eds.), Proceedings of the 
$9^{\text {th }}$ International Conference: Mathematics Education in a Global Community (pp. 160-164). Charlotte, NC: Mathematics in the $21^{\text {st }}$ Century.

Engeström, Y. (1994). The working health center project: Materializing zones of proximal development in a network of organizational learning. En T. Kauppinen, \& M. Lahtonen (Eds.), Action research in Finland (pp. 233-272). Helsinki, Finlandia: Ministry of Labour.

Englund, T. (2006). Deliberative communication: A pragmatist proposal. Journal of Curriculum Studies, 38(5), 503-520.

Freire, P. (1988). Pedagogia do oprimido. Rio de Janeiro, Brasil: Paz e Terra.

Giroux, H., \& McLaren, P. (1989). Critical pedagogy, the state and cultural struggle. Albany, NY: State University of New York Press.

Godino, J. D., Font, V., Wilhelmi, M. R., \& Castro, C. (2009). Aproximación a la dimensión normativa en didáctica de las matemáticas desde un enfoque ontosemiótico. Enseñanza de las Ciencias, 27(1), 59-76.

Habermas, J. (1998). On the pragmatics of communication. Cambridge, MA: MIT Press.

Lantolf, J., Thorne, S. L., \& Poehner, M. (2015). Sociocultural theory and second language development. En B. van Patten, \& J. Williams (Eds.), Theories in second language acquisition (pp. 207-226). Nueva York: Routledge.

Planas, N. (2017). Aprendizaje matemático multilingüe: Qué se sabe y desde qué teorías. En J. M. Muñoz-Escolano, A. Arnal-Bailera, P. Beltrán-Pellicer, M. L. Callejo, \& J. Carrillo (Eds.), Actas del XXI Simposio de la Sociedad Española de Investigación en Educación Matemática (pp. 91-105). Zaragoza: SEIEM.

Planas, N., \& Boukafri, K. (2018). Construcción de normas generadoras de oportunidades para el aprendizaje matemático. En A. Ávila (Ed.), Rutas de la educación matemática (pp. 37-51). Ciudad de México: SOMIDEM.

Planas, N., \& Edo, M. (2008). Dificultades de aprendizaje matemático asociadas al aula multicultural. Boletim do Grupo de Estudos e Pesquisas em Educaçao Matemática, 52, 11-28.

Planas, N., \& Valero, P. (2016). Tracing the socio-cultural-political axis in understanding mathematics education. En Á. Gutiérrez, G. C. Leder, \& P. Boero, The second handbook of the psychology of mathematics education. The journey continues (pp. 447-479). Rotterdam, Holanda: Sense Publishers.

Samuelsson, M. (2016) Education for deliberative democracy A typology of classroom discussions. Democracy and Education, 24(1), 3-9.

Serradó, A., Vanegas, Y., \& Giménez, J. (2015). Facilitating deliberate dialogue in mathematics classroom. En U. Gellert, J. Giménez, C. Hahn, \& S. Kafoussi (Eds.), Educational paths to mathematics (pp. 289-303). Cham, Suiza: Springer.

Skovsmose, O. (1999). Hacia una filosofía de la educación matemática crítica. Bogotá, Colombia: Una empresa docente -Universidad de los Andes.

Skovsmose, O., \& Valero, P. (2002). Democratic access to powerful mathematics in a democratic country. En L. English (Ed.), Handbook of international research in mathematics education (pp. 383-408). Mahwah, NJ: Lawrence Erlbaum Assoc. 
Skovsmose, O., \& Valero, P. (2012). Acceso democrático a ideas matemáticas poderosas. En P. Valero, \& O. Skovsmose (Eds.), Educación matemática critica. Una visión sociopolítica del aprendizaje y la enseñanza de las matemáticas (pp. 25-61) Bogotá, Colombia. Una empresa docente.

Valero, P. (2019). Deliberative mathematics education for social democratization in Latin America. ZDM-Mathematics Education, 99(1), 20-26.

Vanegas, Y. (2013). Competencias ciudadanas y desarrollo profesional en matemáticas. Trabajo de Tesis Doctoral. Universitat de Barcelona, España.

Vanegas, Y., D’Ambrosio, U., \& Giménez, J. (2019). Discurso y prácticas democráticas en la clase de matemáticas. REDIMAT-Journal of Research in Mathematics Education, 8(2), 139-165.

Walshaw, M., \& Anthony, G. (2006). Numeracy reform in New Zealand: Factors that influence classroom enactment. En J. Novotná, H. Moraová, M. Krátká, \& N. Stehlíková (Eds.), Proceedings $30^{\text {th }}$ Conference of the International Group for the Psychology of Mathematics Education (Vol. 5, pp. 361-368). Praga, República Checa: PME.

\section{Referencias de los autores}

Yuly Vanegas, Universitat de Lleida (España).yuly.vanegas@udl.cat

Joaquín Giménez, Universitat de Barcelona (España).quimgimenez@ub.edu 


\title{
Democratic mathematical practices: Analysis of a school experience
}

\author{
Yuly Vanegas, Universitat de Lleida \\ Joaquín Giménez, Universitat de Barcelona
}

In this article we propose and explain the characterization of the democratic mathematical practices emerging in a sequence of mathematics lessons with 12- and 13-year-old students, focused on the teaching and learning of the length measurement. It is shown that the promotion and enactment of such practices in the classroom makes it possible to successfully produce and negotiate mathematical meanings. An analysis is designed and carried out in which, in addition to the identification of democratic mathematical practices, we observe norms to describe and interpret the interactional processes that shape the co-participation of teacher and students throughout the enactment of mathematical practices in the lessons. Findings indicate a predominance of practices related to the articulation and resolution of conflicts of a diversity of origins and with numerous meanings implied. It is also found that each type of democratic mathematical practice relates to a certain type of norm that regulates the continuation or even the activation of the corresponding practice. All these major findings are illustrated by means of a concrete short episode in one of the lessons where a variety of types of practice and of types of norm can be easily inferred, as well as some of the relationships between both types. Our theoretical tools are taken from social theories of mathematics education and learning put together with the analytical construct of norm as it has been developed within the ontosemiotic aprproach in the context of the broader research field. Overall, our study contributes to the domain of classroom research on mathematics teaching and learning in which the promotion of democratic values, critical citizenship, and social justice is an explicit aim. 